R.M. Hanna, MD

S.E. Marsh, MS

D. Swistun, MS

L. Al-Gazali, MB, ChB, MRCP

M.S. Zaki, MD, PhD

G.M. Abdel-Salam, MD, $\mathrm{PhD}$

A. Al-Tawari, MD

L. Bastaki, MD

H. Kayserili, MD

A. Rajab, MD, DCH, MRCP, PhD

B. Boglárka, MD

R.B. Dietrich, MD

W.B. Dobyns, MD

C.L. Truwit, MD

S. Sattar, MD

N.A. Chuang, MD

E.H. Sherr, MD, PhD

J.G. Gleeson, MD

Address correspondence and reprint requests to Dr. J.G. Gleeson, LBR 462, 9500 Gilman Dr., La Jolla, CA 92093-0665 jogleeson@ucsd.edu

Supplemental data at www.neurology.org

\section{Distinguishing 3 classes of corpus callosal abnormalities in consanguineous families}

\section{ABSTRACT}

Objective: We sought to create a classification system for pediatric corpus callosal abnormalities (CCA) based upon midline sagittal brain MRI. We used the term CCA for patients with structural variants of the corpus callosum, excluding patients with interhemispheric cyst variant or pure dysplasia without hypoplasia. Currently, no system exists for nonsyndromic forms of CCA, and attempts to create such a system have been hampered by highly variable morphology in patients with sporadic CCA. We reasoned that any useful strategy should classify affected family members within the same type, and that phenotypic variability should be minimized in patients with recessive disease.

Methods: We focused recruitment toward multiplex consanguineous families, ascertained 30 patients from 19 consanguineous families, and analyzed clinical features together with brain imaging.

Results: We identified 3 major CCA classes, including hypoplasia, hypoplasia with dysplasia, and complete agenesis. Affected individuals within a given multiplex family usually displayed the same variant of the class of abnormality and they always displayed the same class of abnormality within each family, or they displayed complete agenesis. The system was validated among a second cohort of 10 sporadic patients with CCA.

Conclusions: The data suggest that complete agenesis may be a common end-phenotype, and implicate multiple overlapping pathways in the etiology of CCA. Neurology ${ }^{\circledR} 2011 ; 76: 373-382$

\section{GLOSSARY}

AC $=$ anterior commissure; $\mathbf{A C C}=$ agenesis of the corpus callosum; $\mathbf{C C}=$ corpus callosum; $\mathbf{C C A}=$ corpus callosal abnormalities; $\mathbf{G W}=$ weeks gestation; $\mathbf{P B}=$ Probst bundle; $\mathbf{U A E}=$ United Arab Emirates.

The corpus callosum (CC) structurally consists of 4 major anatomic features including the rostrum, genu, corpus, and splenium (figure 1). Corpus callosal abnormalities (CCA), a term encompassing agenesis of the corpus callosum (ACC), has an estimated prevalence of $0.3 \%-$ $0.7 \%$ in patients undergoing brain imaging, ${ }^{1}$ but in Middle Eastern populations the prevalence is probably closer to $1 \%,{ }^{2,3}$ due to the higher rates of inbreeding. The finding of CCA can be identified incidentally, or can be part of a developmental or cytogenetic disease.

Previous studies have differentiated CCA into those with or without additional brain malformations ${ }^{3}$ or into partial or complete agenesis. ${ }^{4}$ However, in one study, roughly equal percentages of patients with either partial or complete agenesis displayed additional structural brain abnormalities, suggesting that this differentiation may not relate to causative etiology. ${ }^{5}$ The presence vs absence of Probst bundles (PBs) has also been used to define categories of disease, ${ }^{3}$

\footnotetext{
From the Department of Neurosciences and Pediatrics (R.M.H., S.E.M., D.S., S.S., J.G.G.), Rady Children's Hospital, Howard Hughes Medical Institute, San Diego, CA; Department of Paediatrics (L.A.-G.), Faculty of Medicine and Health Sciences, United Arab Emirates University, Al Ain, United Arab Emirates; Clinical Genetics Department (M.S.Z., G.M.A.-S.), Human Genetics and Genome Research Division, National Research Centre, Cairo, Egypt; Pediatric Neurology Unit (A.A.-T.), Al Sabah Hospital, Kuwait Medical Genetics Centre (L.B.), Kuwait City, Kuwait; Medical Genetics Department (H.K.), Istanbul Medical Faculty, Istanbul University, Istanbul, Turkey; Genetic Unit (A.R.), Ministry of Health, Muscat, Sultanate of Oman; Department of Pediatrics (B.B.), Semmelweis University, Budapest, Hungary; Department of Radiology (R.B.D.), University of California San Diego; Departments of Pediatrics and Neurology (W.B.D.), University of Washington, Seattle; Department of Radiology, Neuroradiology, and Magnetic Resonance (C.L.T.), University of Minnesota School of Medicine, Minneapolis; Neuroradiology Division (N.A.C.), Rady Children's Hospital, San Diego Imaging Medical Group, San Diego, CA; and Department of Neurology (E.H.S.), University of California, San Francisco.

Study funding: Supported by the NIH (K24 NS057382 and R01 NS NS48452 to J.G.G.).

Disclosure: Author disclosures are provided at the end of the article.
} 
Figure 1 Classification of morphologic spectrum of midline sagittal MRI appearance of the corpus callosum (CC)

Schematic representation NORMAL

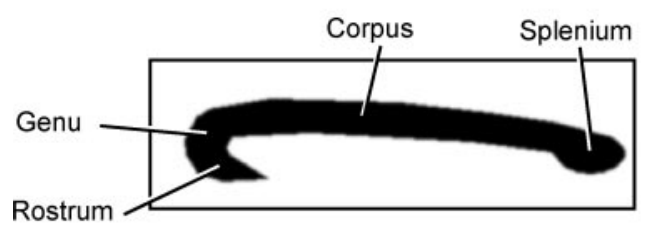

HYPOPLASIA

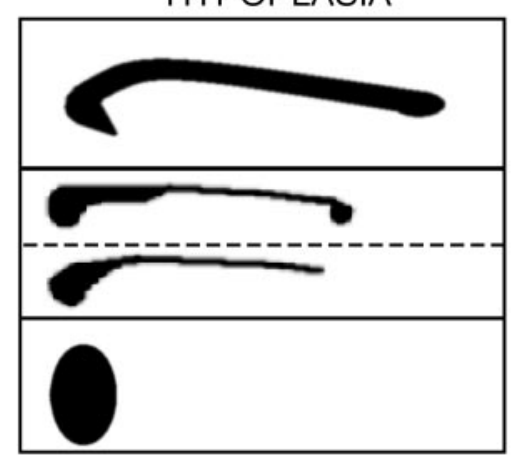

DYSPLASIA

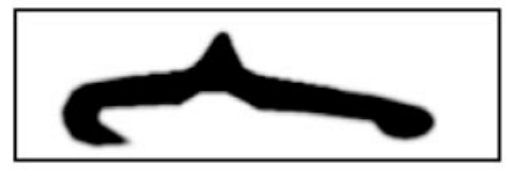

HYPOPLASIA with DYSPLASIA

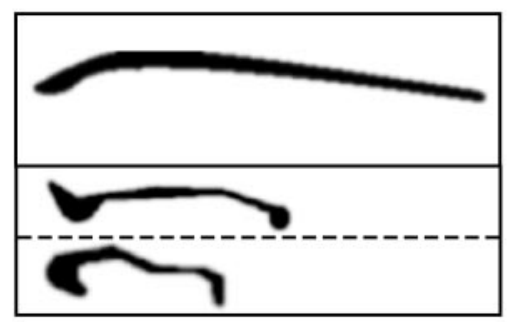

COMPLETE AGENESIS

\section{Hallmarks}

Characteristic shape and size

\section{Hypoplasia without dysplasia Generalized hypoplasia but intact morphology}

Apple core CCA Hypoplasia of posterior CC

\section{Anterior remnant CCA Agenesis of the mid and posterior $\mathrm{CC}$ with an} anterior remnant

Hump-shaped CC

Dysplasia without hypoplasia Distinct from CCA

\section{Stripe CCA}

Uniformly thinned

$\mathrm{CC}$, with dysplasia

\section{Kinked CCA \\ Hypoplasia \\ and kinked CC}

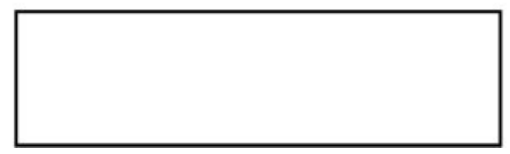

\section{Complete agenesis \\ Completely absent CC at the resolution of MRI}

Schematics depict the appearance of CC on midline sagittal MRI. Appearance of normal CC showing rostrum, genu, corpus, and splenium. CC abnormalities were divided into principal classes: HYPOPLASIA, DYSPLASIA WITHOUT HYPOPLASIA, HYPOPLASIA WITH DYSPLASIA, and COMPLETE AGENESIS. There are 3 subtypes of HYPOPLASIA: hypoplasia without dysplasia, apple core corpus callosal abnormalities (CCA), and anterior remnant CCA, based upon the physical appearance. DYSPLASIA WITHOUT HYPOPLASIA encompasses cases in which the CC is morphologically abnormal but has no evidence of hypoplasia, and is represented by a case with hump-shaped CC. ${ }^{25}$ In HYPOPLASIA WITH DYSPLASIA CCA, there are 2 subtypes, stripe CCA and kinked CCA. COMPLETE AGENESIS is characterized by absence of an evident CC at the level of MRI. 
Total $=12$
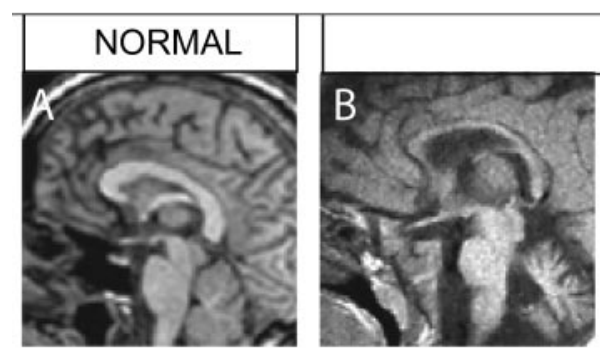

$\mathrm{n}=6$

Hypoplasia without dysplasia CCA
HYPOPLASIA

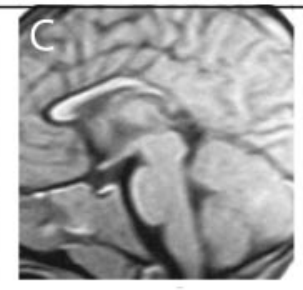

$\mathrm{n}=5$

Apple core CCA

(1)

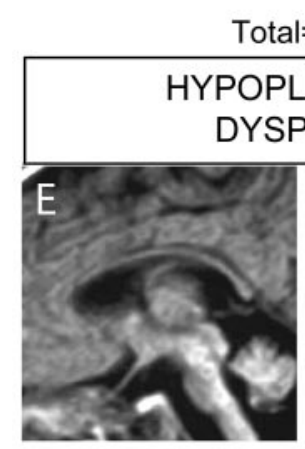

$\mathrm{n}=6$

Stripe CCA
Total=12

POPLASIA with

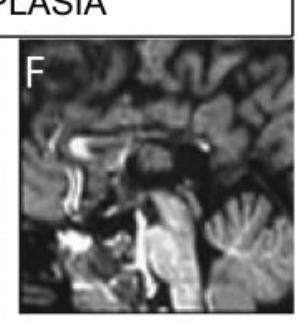

$\mathrm{n}=6$

Kinked CCA

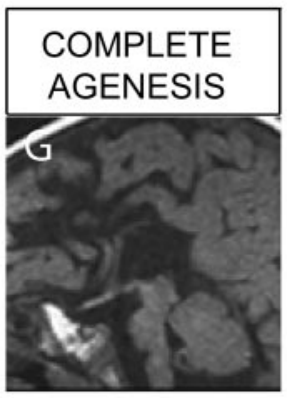

$\mathrm{n}=5$

Complete agenesis

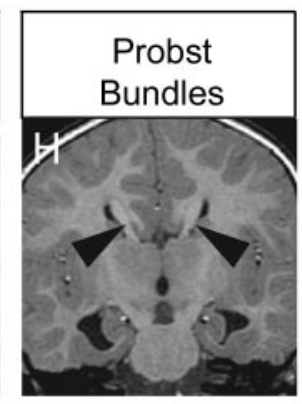

$n=1$

(A) Normal CC. (B-D) HYPOPLASIA class of CCA. (B) Hypoplasia without dysplasia CCA, (C) apple core CCA, (D) Anterior remnant CCA (black arrow). (E, F) HYPOPLASIA WITH DYSPLASIA class of CCA. (E) Stripe CCA, (F) kinked CCA. (G) COMPLETE AGENESIS. (H) Example of Probst bundles. Number of patients with each class of CCA, and each subtype of CCA, from a cohort of 29 patients.

losal dysplasia7 (figure 1) and ACC with interhemispheric cyst $^{8}$ were not encountered and are thus not discussed further.

We identified 3 principal classes of CCA among our cohort (figures 1 and 2), all designated with upper case typeface. Within some classes, there were 2 or more variants, designated with italic typeface. HYPOPLASIA consisted of uniformly thin or partially underdeveloped CCA in the posterior region. Within the HYPOPLASIA class, we identified hypoplasia without dysplasia CCA, with all major anatomic features intact including a distinctive rostrum, genu, corpus, and splenium. More severe forms of HYPOPLASIA showed progressive loss of the more caudal aspects of the CC. This includes a form where the posterior CC is hypoplastic and foreshortened (i.e., corpus or splenium), taking on the appearance of an apple core CCA, and a form in which there is agenesis of the posterior CC (i.e., corpus and splenium), called anterior remnant CCA type.

The second class of CCA was defined as HYPOPLASIA WITH DYSPLASIA, with distur- bance of the overall shape of the CC that was not limited to the posterior region, with 2 subtypes. The first consisted of a uniformly thin stripe CCA that lacked an anatomically distinct genu and splenium. The second was characterized by a thin CC that was hypoplastic as well as obviously kinked at one or more locations, either anteriorly or posteriorly, which we called kinked CCA.

The third class of CCA was characterized by absent CC at the resolution of MRI on a midline sagittal image. This class would thus be synonymous with COMPLETE AGENESIS or the previously used term ACC. In this class, it was important to distinguish the hippocampal commissure from the CC, the former retained in many COMPLETE AGENESIS cases. $^{6}$

We also specifically commented on the appearance of the anterior commissure (AC), because recent data suggest a compensatory increase in the size of the AC (or other structures such as the internal capsule) in some cases of ACC. ${ }^{9}$ The AC was normal is 7 , decreased in size in 14 , absent in 8 , and enlarged in 
Table 1 Clinical features of patients with CCA in this study

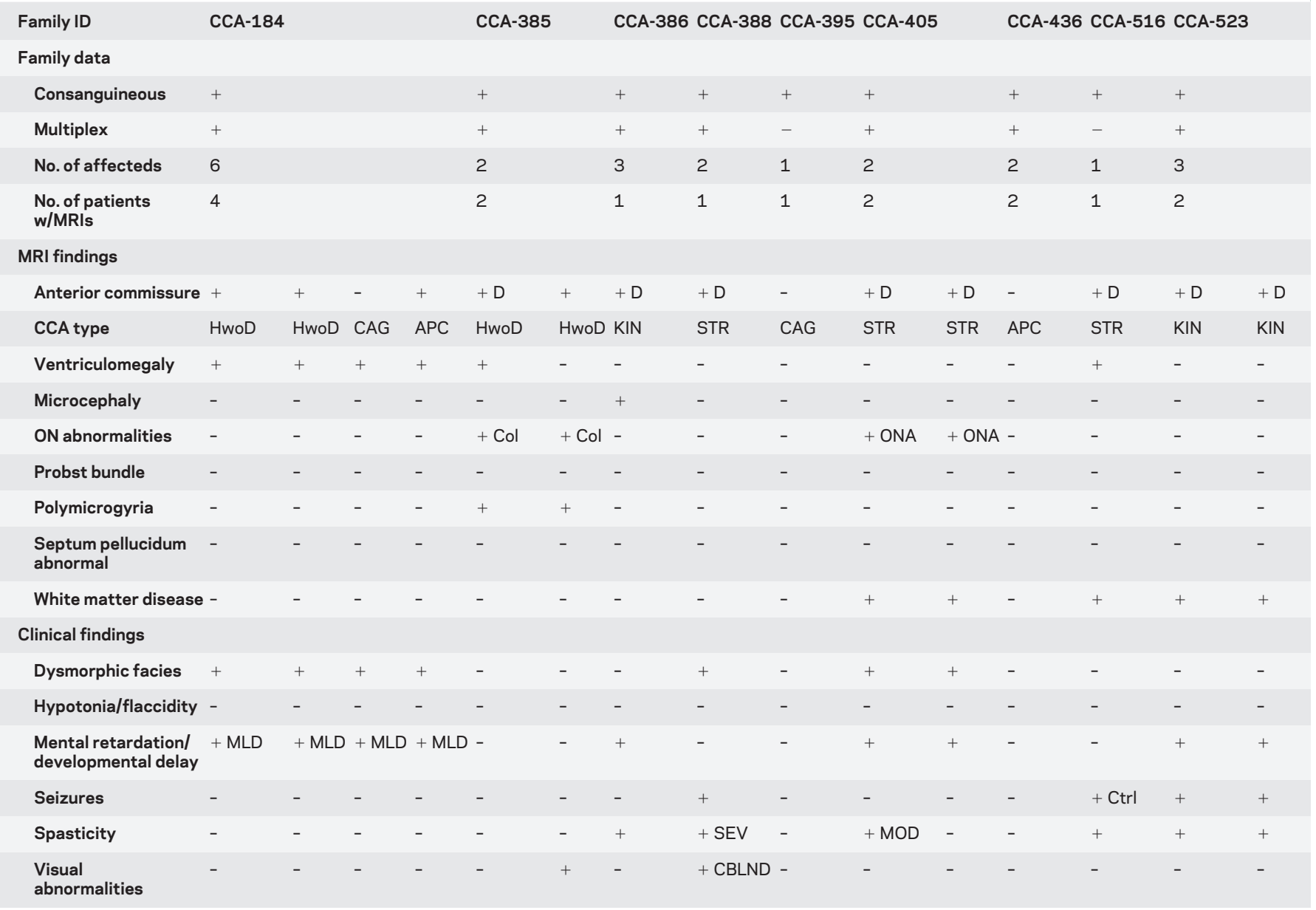

-Continued

Abbreviations: $+=$ present; $-=$ absent $;+D=$ decreased size: referring to anterior commissure); $+\mathrm{E}=$ enlarged size: referring to anterior commissure); ABS = seizures (absence type); ANR = anterior remnant corpus callosum; APC = apple core corpus callosum; $C A G=$ complete absence of corpus callosum; CBLND = cortical blindness; CCA = corpus callosum abnormality; Col = optic colobomas; CSP = cavum septum pellucidum; Ctrl = controlled (referring to seizures); $\mathrm{HwoD}=$ hypoplasia without dysplasia; $\mathrm{KIN}=$ kinked corpus callosum; $\mathrm{MLD}=$ mild; $\mathrm{NCtrl}=$ not controlled (referring to seizures); $\mathrm{ON}=$ optic nerve; ONA = optic nerve atrophy; PS = perisylvian; SEV = severe; STR = stripe corpus callosum; VEP = visual evoked potentials.

1 (table 1; see table e-1 on the Neurology ${ }^{\circledR}$ Web site at www.neurology.org for additional details). We did not utilize the appearance of the AC in our classification system due to unavoidable limitations in MRI quality in several cases.

We categorized the 30 patients based upon this simple classification system (figure 2). We found 13 patients with HYPOPLASIA appearance, and of these, 6 showed hypoplasia without dysplasia CCA, 5 showed apple core CCA, and 2 showed anterior remnant $C C A$ appearance. We found 12 patients with HYPOPLASIA WITH DYSPLASIA class, and of these, 6 showed stripe CCA and 6 showed a kinked $C C A$ appearance. There were 5 patients with COMPLETE AGENESIS.

Validation of classification system in multiplex consanguineous families. CCA-184, multiple epiphyseal dysplasia, and CCA. CCA-184 is a consanguineous multiplex family with 3 affected branches from the
United Arab Emirates (UAE, table 1 and figure 3). The affecteds within this family also displayed a mild form of multiple epiphyseal dysplasia, described previously, ${ }^{10,11}$ mild mental retardation, developmental delay, and ventriculomegaly. MRI was available in 4 of the affected members, and 2 (IV-I-2, IV-I-4) showed an intact splenium and genu, and were thus categorized as hypoplasia without dysplasia. In IV-II-7, the CCA was classified as the subtype apple core CCA because of foreshortening. In IV-III-1, there were fetal ultrasound studies that showed similar features, but MRI was not available, so he was excluded from further analysis. Interestingly, in IV-I-9, MRI showed no discernible CC, consistent with COMPLETE AGENESIS.

CCA-385, cerebellar vermis hypoplasia, and CCA. Family CCA-385 displayed 2 affected branches and is from the UAE (figure 3). The clinical phenotype includes $\mathrm{GnRH}$ deficiency with pituitary hypoplasia, 


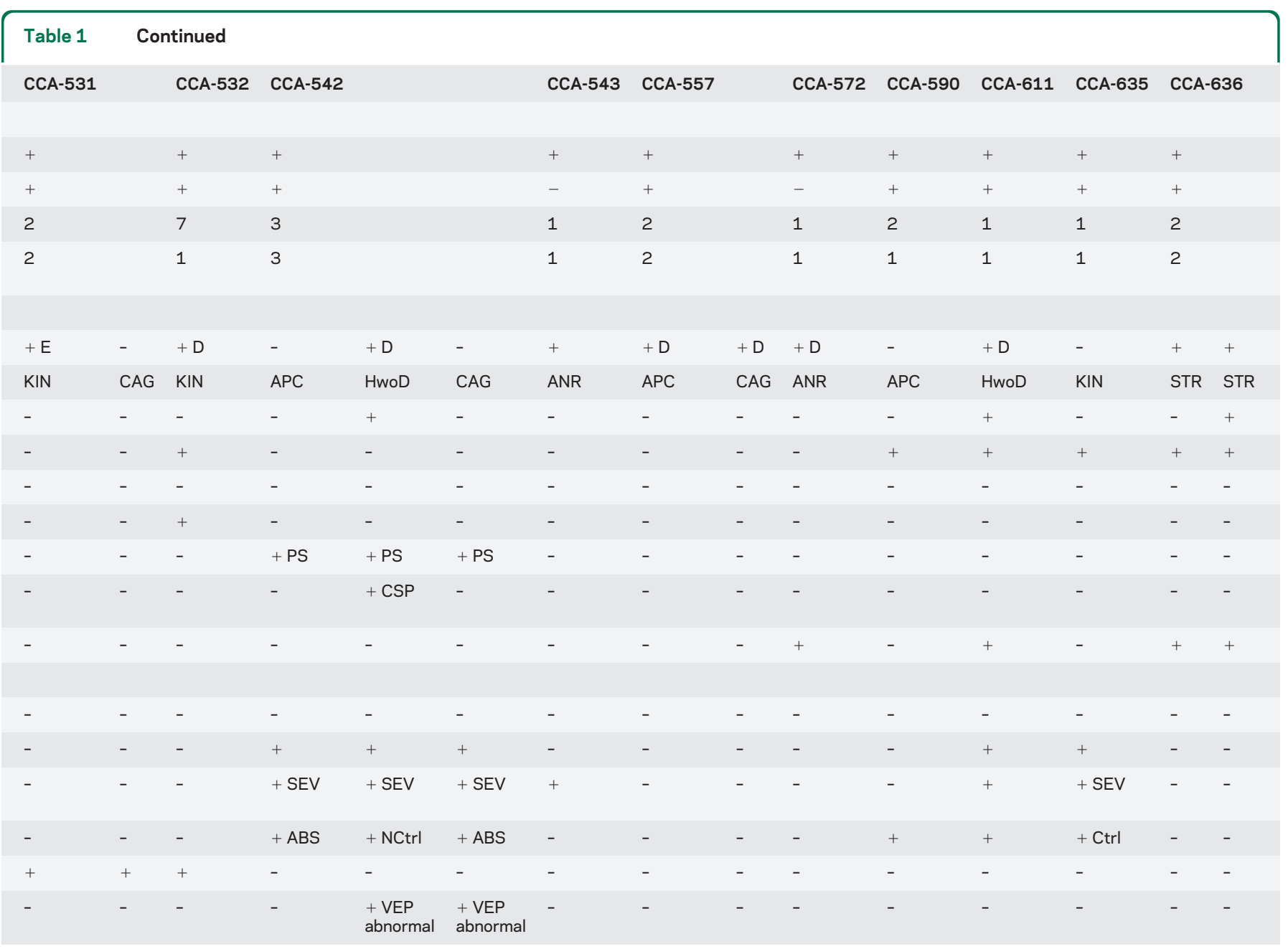

visual reduction, and mild ichthyosis. ${ }^{12}$ The affected members (IV-I-5, IV-II-7) showed mild ventriculomegaly, cerebellar vermis hypoplasia, and perisylvian polymicrogyria. The CC showed thinning of the entire structure, with most notable hypoplasia of the mid to posterior corpus, with an intact genu and splenium, consistent with hypoplasia without dysplasia CCA. MRI was not available on IV-II-5.

CCA-542, epilepsy, hypotonia, hyporeflexia, and CCA. Family CCA-542 has one affected branch from a first-cousin marriage and is from Kuwait (figure 3). Their clinical phenotype included epilepsy, hypotonia, hyporeflexia, ventriculomegaly, and visual reduction. All 3 affected members showed perisylvian polymicrogyria. In III-3 the MRI showed posterior shortening of the CC with underdeveloped or absent splenium, consistent with apple core CCA. III-5 CC was classified as hypoplasia without dysplasia CCA. In III-9, MRI showed

\section{COMPLETE AGENESIS.}

CCA-557, pontocerebellar hypoplasia, and CCA. Family CCA-557 displays 2 affected members from a first-cousin marriage from Turkey (figure 3). The clinical phenotype suggested pontocerebellar hypoplasia. MRI in III-1 showed thinning and foreshortening of the CC, consistent with apple core CCA subtype. In III-2, MRI showed COMPLETE AGENESIS.

CCA-405, mental retardation, dysmorphic facies, and CCA. Family CCA-405 displays 2 affected members from a first-cousin marriage from Oman (figure 4). Their clinical phenotype included mental retardation, dysmorphic facial features, and moderate spasticity in one of two children. MRI in III-1 and III-2 showed evidence of white matter disease with high signal on T2 and fluid-attenuated inversion recovery images and optic nerve atrophy. Lysosomal storage enzyme analysis, serum amino acids, urine organic 
CCA-184

HYPOPLASIA/COMPLETE AGENESIS

Hypoplasia without dysplasia/Apple core/Complete agenesis

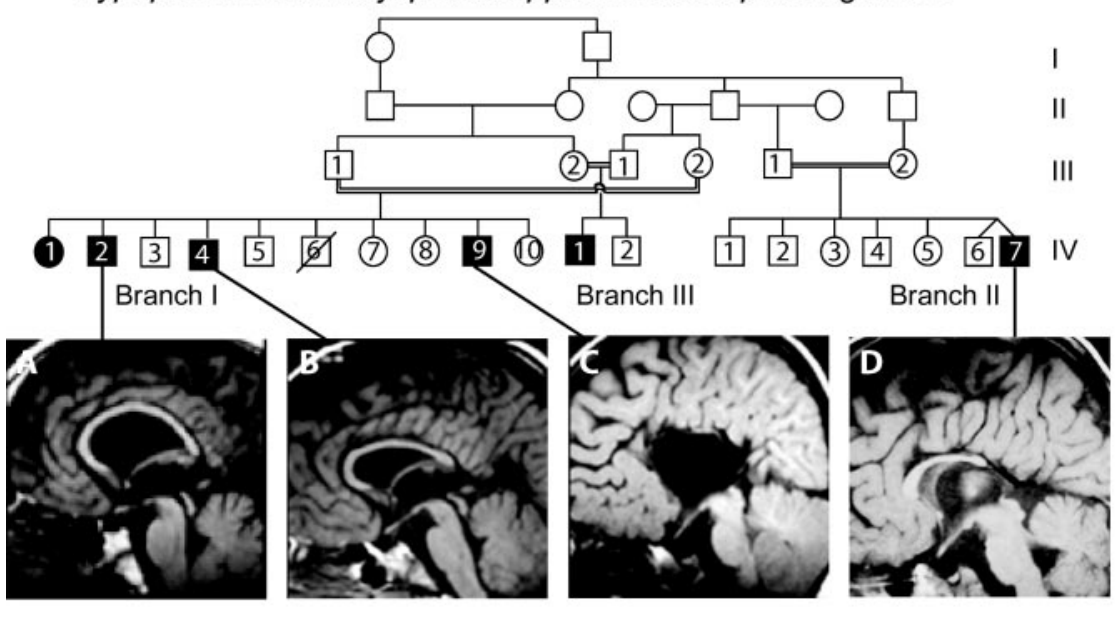

CCA-542

HYPOPLASIA/COMPLETE AGENESIS

Hypoplasia without dysplasia/Apple core/Complete agenesis

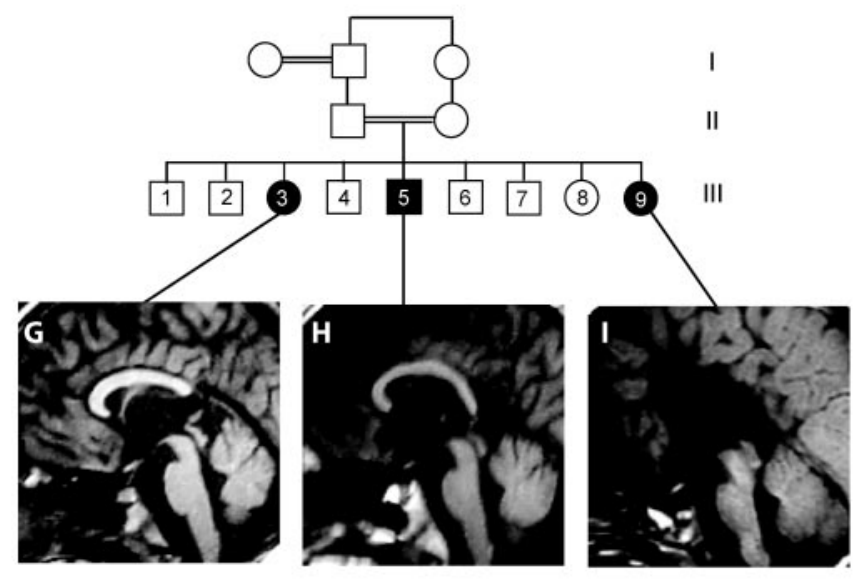

CCA-385

HYPOPLASIA

Hypoplasia without dysplasia

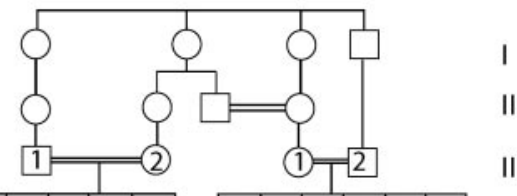

(1) (3) 45612 (3) 4587 IV

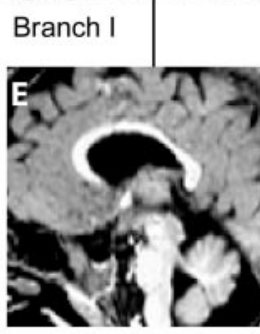

Branch II

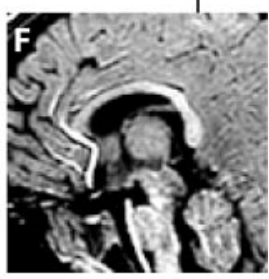

CCA-557

HYPOPLASIA/COMPLETE AGENESIS

Apple core CCA/Complete agenesis

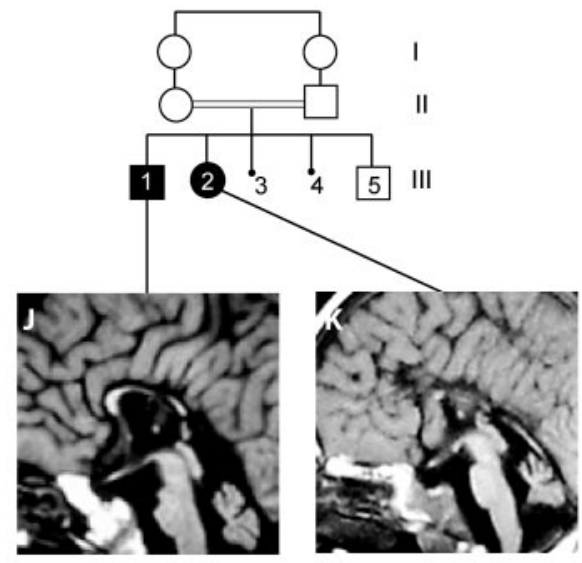

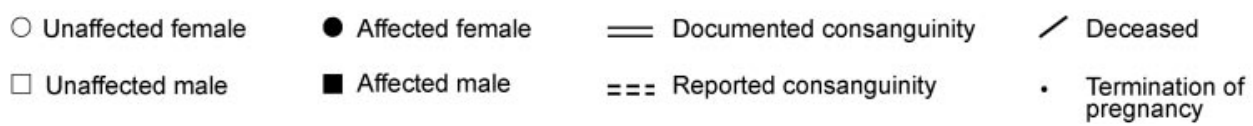

(Top row) CCA-184: Affected children have multiple epiphyseal dysplasia, mild mental retardation, developmental delay, prominent ventricles, and corpus callosal abnormalities (CCA). (A, B) HYPOPLASIA, hypoplasia without dysplasia CCA. (D) HYPOPLASIA, apple core CCA subtype. (C) COMPLETE AGENESIS. CCA-385: Affected children have GnRH deficiency, respiratory abnormalities, septo-optic dysplasia, bilateral optic colobomas, pituitary hypoplasia, cerebellar vermis hypoplasia, mild polymicrogyria, and CCA. (E, F) Hypoplasia without dysplasia CCA. (Bottom row) CCA542: Affected children have epilepsy, hypotonia and hyporeflexia, perisylvian polymicrogyria, and CCA. (G) HYPOPLASIA, apple core CCA. (H) HYPOPLASIA, Hypoplasia without dysplasia CCA. (I) COMPLETE AGENESIS. CCA-557: Affected children have cerebellar vermis hypoplasia and CCA. (J) Apple core CCA. (K) COMPLETE AGENESIS.

acids, as well as lactate and pyruvate studies were unremarkable, and the clinical status improved over time. There was a uniformly thin appearance of the CC, consistent with stripe CCA, without clear anatomic features of a genu or splenium.

CCA-636, pontocerebellar hypoplasia, microcephaly, and CCA. This family has 2 affected members from a firstcousin marriage from Egypt (figure 4). The clinical phenotype includes mild microcephaly, mild hyp- oplasia of the pons and cerebellum, and mild ventriculomegaly. MRI in III-1 and III-2 showed mild ventriculomegaly and a corpus callosum without a discernible genu, body, isthmus, and splenium that was classified as stripe CCA.

CCA-523, mental retardation, epilepsy, and CCA. Family CCA-523 displays 2 affected members from a first-cousin (once removed) marriage, from Egypt (figure 4). Their clinical phenotype included mental 
CCA-405

HYPOPLASIA WITH DYSPLASIA

Stripe CCA

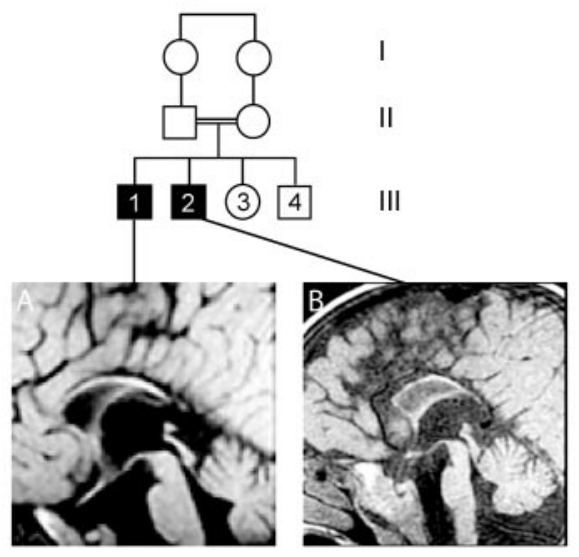

CCA-636

HYPOPLASIA WITH DYSPLASIA

Stripe CCA

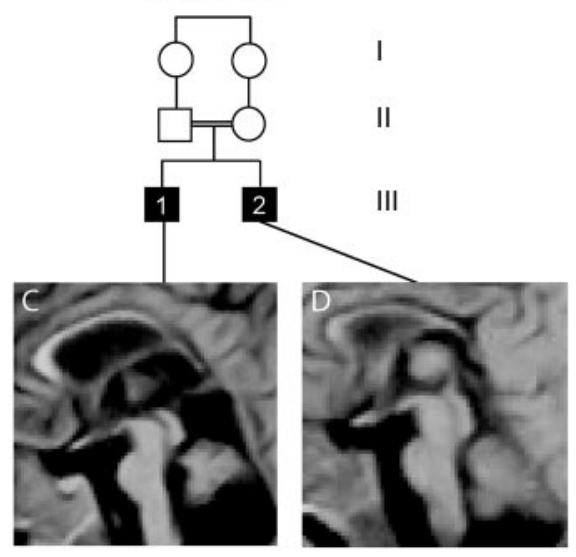

CCA-523

HYPOPLASIA WITH DYSPLASIA

Kinked CC

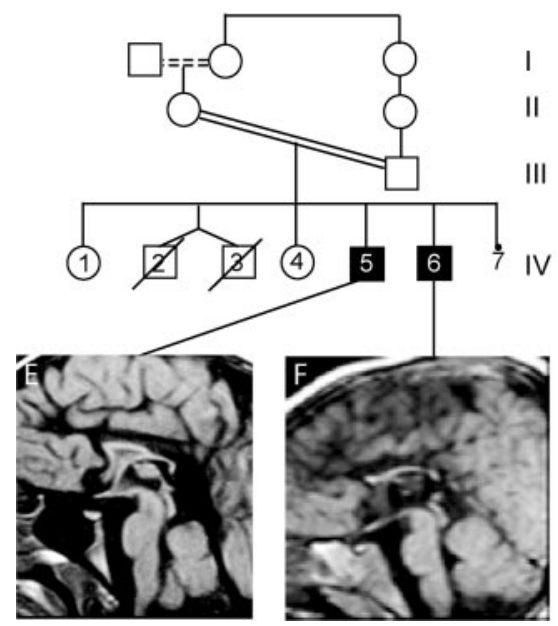

CCA-531

HYPOPLASIA WITH DYSPLASIA/COMPLETE AGENESIS Kinked CCA/Complete agenesis

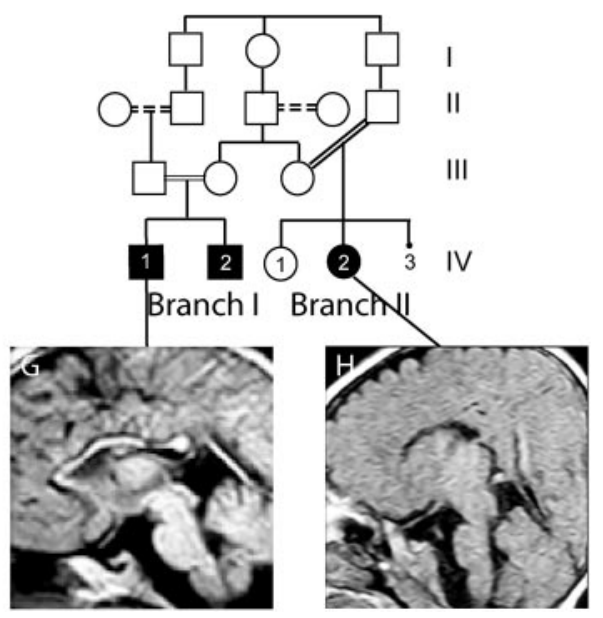

(Top row) CCA-405: Affected children have mental retardation, dysmorphic features, and corpus callosal abnormalities (CCA). (A, B) Stripe CCA. CCA-636: Affected children have spasticity, ataxia, white matter hypoplasia, cerebellar hypoplasia, and CCA. (C, D) Stripe CCA. (Bottom row) CCA-523: Affected children have spasticity, epilepsy, and CCA. (E, F) Kinked CCA. CCA-531: Affected children have cerebellar vermis hypoplasia, spasticity, and CCA. (G) Kinked CCA. (H) COMPLETE AGENESIS.

retardation, spasticity, and epilepsy. The MRI showed similar appearance in the 2 affected members, the CC was severely thinned and kinked both in the anterior and posterior parts, most consistent with kinked CCA subtype. Additionally, there was some evidence of abnormal signal in the white matter, and was slightly more severe in IV-6.

CCA-531, cerebellar vermis hypoplasia, spasticity, and CCA. Family CCA-531 displays 2 affected branches from Egypt (figure 4). There are 3 affected siblings that resulted from either a second-cousin or a firstcousin (once removed) marriage. MRI data were available from 2 affecteds. Their clinical phenotype included mild spasticity and microcephaly. In IVI-1, MRI showed a thin CC that was kinked near the anterior region and the isthmus, consistent with kinked CCA subtype. In IV-II-2 MRI at 2 years showed complete absence of the CC, consistent with COMPLETE AGENESIS.

Evaluation of classification system in a cohort of CCA probands from consanguineous families. In order to determine if the proposed classification system could be applied to other cases with CCA, we used the system to classify an additional 10 families with CCA from our database (CCA-386, CCA-388, CCA-395, 
CCA-436, CCA-516, CCA-532, CCA-543, CCA572, CCA-590, CCA-611, and CCA-635). All reported a history of consanguinity (typically firstcousin marriage), and 7 are multiplex (with more than one affected child), but in each case MRI was available from only a single affected within the family. There were 5 patients with some form of the HYPOPLASIA class, and of these, 2 patients showed hypoplasia without dysplasia CCA, 2 showed apple core $C C A$, and one showed anterior remnant $C C A$ appearance (figure e-1). There were 5 patients with one of the HYPOPLASIA WITH DYSPLASIA class, and of these, 2 showed stripe CCA and 3 showed kinked CCA. There was one patient with COMPLETE AGENESIS. Therefore, the classification system appears to be applicable to patients with various forms of CCA, at least within the cohort tested.

Validation of the classification system by a blinded neuroradiologist and neurologist. In order to validate the classification system proposed here, we presented the unlabeled images to both a blinded neuroradiologist and neurologist, each separately categorizing each patient according to the proposed classification system. As a result, there were 29 scans evaluated and concordance was established in 26 (89\%) and 25 (86\%), which is similar reliability to the $80 \%-90 \%$ concordance rate of other neuroimaging classification systems. ${ }^{13,14}$ The scans in which there was nonconcordance were at the interface of the hypoplasia without dysplasia and stripe CCA, because these 2 are along a continuum without an absolutely distinct separating line.

Clinical data. Five of the 6 hypoplasia without dysplasia CCA cases were associated with mild to moderate ventriculomegaly $(83 \%)$, whereas there was only one apple core CCA out of the 5, and 2 striped CCA out of the 6 that were associated with ventriculomegaly (table 1). Fifteen patients with CCA also had cerebellar affection of varying degrees (50\%) and 7 (23\%) had microcephaly (OFC $<2 \%$ ile), but no correlation with type of CCA was apparent. There were 9 patients in our cohort with reduced white matter or evidence of abnormal white matter signal (30\%), but this was observed in both HYPOPLASIA and HYPOPLASIA WITH DYSPLASIA classes. There were no obvious correlations between the presence or severity of extra-CNS or clinical features with the subtype or severity of CCA that we could detect. Statistical analysis was not attempted secondary to the small size and highly diverse phenotypic spectrum of the patient cohort.

DISCUSSION The proposed CCA classification system can be applied when evaluating a single midline sagittal MRI from any patient, and does not require 3-dimensional reconstruction or volumetric analysis. Although future studies might utilize more advanced imaging technologies such as fMRI or fractional anisotropy for more highly precise anatomic subclassification, for most clinicians, a simple system based upon routine MRI is a necessary first step. We found that within a given family, the appearance of the CCA was remarkably similar, though in some instances there were different subtypes of a given class of abnormality seen within the same family. Clearly there is some interfamilial variability, although COMPLETE AGENESIS can be observed in family members as an "end phenotype" associated with most of the subtypes. Due to the relatively small cohort size, statistical analysis could not be applied, and we could not determine whether particular CNS or non-CNS clinical features consistently associated with specific CCA subtype.

The sequence of CC development begins with the differentiation of the commissural plate around 6 weeks gestation (GW), followed by crossing of pioneer axons, first in the rostrum and then proceeding more casually from 10 to 12 GW. ${ }^{9}$ CC myelination does not occur until after birth and proceeds until adolescence. Given this sequence, we might imagine that the insult timing of apple core and anterior remnant might be prior to midgestation, whereas hypoplasia without dysplasia might be later. Pinpointing genetic etiologies could better clarify precise mechanisms.

The clinical phenotype tended to be similar in siblings of a given family, but there was greater heterogeneity than for MRI findings. Some of the patients had evidence of a small brainstem/pons, which might represent deficient longitudinal pontine axons as part of an overall axonal deficiency in CCA, but a more comprehensive volumetric analysis of brainstem was not possible in this cohort due to limited image availability. Previous studies comparing neurodevelopmental outcome in relationship to partial or complete CCA have shown mixed results, some finding that complete CCA shows a poorer prognosis, ${ }^{15}$ whereas others have not validated this correlation. ${ }^{4,16}$ We found that the $\mathrm{AC}$ was present in most cases; however, in cases with COMPLETE AGENESIS, it tended to be absent or decreased in size.

Our observation fits well with the current understanding of CC development as a process that takes place along distinct anatomic axes, and a number of early events that are guided by independent signaling systems for each anatomic group of axons that cross the midline. ${ }^{17,18}$ Disturbance of an essential early event might therefore lead to hypoplasia or to complete agenesis, depending upon environmental or 
modifier gene influences. The predominant type of HYPOPLASIA WITHOUT DYSPLASIA showed some level of posterior CC hypoplasia, while only 2 cases showed near complete posterior CC hypoplasia (the anterior remnant CCA type). Presumptively in anterior remnant $C C A$ patients, the genetic defect alters neurodevelopmental processes at an early time point, and involves the coordination of axons crossing in all but the most anterior regions of the CC.

A number of genetic disorders in humans have been associated with CCA, including several $\mathrm{X}$-linked diseases, metabolic disorders, and contiguous gene deletion syndromes. ${ }^{17}$ However, in each of these, the CCA is usually secondary to a more defining characteristic (such as in X-linked lissencephaly with ambiguous genitalia syndrome ${ }^{19}$ ), or is just one of a number of defining characteristics (such as in Aicardi or Andermann syndromes). ${ }^{20,21}$ To date, no genes have been identified in which CCA has been the primary defining characteristic. A classification system such as the one that we propose may help lead to the identification of causes of this important neurologic disorder, by allowing patients with the same type of CCA to be grouped together for genetic analysis.

Investigations have identified several potential gene dose-dependent candidate loci for CCA. ${ }^{22-24}$ Additionally, cytogenetic abnormalities are detected in approximately $10 \%$ of patients with CCA. ${ }^{1}$ Analysis in mice has uncovered a large number of genes that when mutated lead to CCA, including guidance molecules, transcription factors, extracellular matrix molecules, signaling/cytoplasmic molecules, growth factors, as well as certain strains of mice that are particularly vulnerable to CCA. ${ }^{17}$ Thus, a large number of genes contribute to the development, morphology, and maintenance of the CC, some of which appear to play primary roles, while in others the effect on the CC may be secondary to a more notable structural CNS defect.

Our preliminary genetic mapping data from these consanguineous families suggest a multitude of new causative loci, which reflects the anatomic complexity that we describe here. Using a recessive disease model, it is possible to identify causative genes using homozygosity mapping and next-generation sequencing strategies in a straightforward manner. Therefore, the ability to select patients for evaluation at newly identified genes based upon similarities in the appearance of the CC will improve the ability to molecularly classify CCA patients.

Given this substantial proportion of sporadic patients with CCA and PBs, we felt our classification scheme should reflect their presence or absence, but rather than further subdivide the aforementioned types, we propose to indicate the presence of $\mathrm{PB}$ s by denoting + PBs next to the type. The explanation for the relatively low percentage of patients with PBs in our cohort compared with historic controls 5 is not immediately apparent but may relate to the bias for large consanguineous families in this study.

\section{ACKNOWLEDGMENT}

The authors thank the collaborators and families who participated in this study and A. James Barkovich for reviewing the text.

\section{DISCLOSURE}

Dr. Hanna, Ms. Marsh, Ms. Swistun, Prof. Al-Gazali, Prof. Zaki, Dr. Abdel-Salam, Dr. Al-Tawari, and Dr. Bastaki report no disclosures. Dr. Kayserili serves on the editorial board of the European Journal of Medical Genetics; serves as a consultant in a clinical genetics outpatient division (10\% effort) and conducts cranial MRI evaluations (20\% effort); and receives research support from the Turkish Research Council, the EU, and the Istanbul University Research Fund. Dr. Rajab and Dr. Boglárka report no disclosures. Dr. Dietrich serves on the editorial board of Investigative Radiology. Dr. Dobyns serves on the editorial boards of the American Journal of Medical Genetics and Clinical Dysmorphology; and receives research support from the NIH. Dr. Truwit serves on the editorial board of Seminars in Roentgenology; is listed as an inventor on multiple patents, mostly related to medical device technologies; receives royalty payments for MR guided neurosurgery devices; receives research support from the $\mathrm{NIH}$; and has served as an expert witness in the field of imaging of perinatal asphyxia. Dr. Sherr receives research support from Pfizer Inc, the NIH/ NINDS, the March of Dimes, the Aicardi Syndrome Foundation, the Weston Havens Foundation, and the National Organization of Disorders of the Corpus Callosum. Dr. Gleeson reports no disclosures.

Received August 4, 2009. Accepted in final form September 28, 2010.

\section{REFERENCES}

1. Bedeschi MF, Bonaglia MC, Grasso R, et al. Agenesis of the corpus callosum: clinical and genetic study in 63 young patients. Pediatr Neurol 2006;34:186-193

2. Chacko A, Koul R, Sankhla DK. Corpus callosum agenesis. Saudi Med J 2001;22:22-25.

3. Sztriha L. Spectrum of corpus callosum agenesis. Pediatr Neurol 2005;32:94-101.

4. Volpe P, Paladini D, Resta M, et al. Characteristics, associations and outcome of partial agenesis of the corpus callosum in the fetus. Ultrasound Obstet Gynecol 2006;27: 509-516.

5. Hetts SW, Sherr EH, Chao S, Gobuty S, Barkovich AJ. Anomalies of the corpus callosum: an MR analysis of the phenotypic spectrum of associated malformations. AJR Am J Roentgenol 2006;187:1343-1348.

6. Barkovich AJ. Pediatric Neuroimaging. Philadelphia: Lippincott Williams \& Wilkins; 2005.

7. Erdogmus B, Yazici B, Ozdere BA. Hump-shaped corpus callosum. Acta Radiol 2005;46:616-617.

8. Barkovich AJ, Simon EM, Walsh CA. Callosal agenesis with cyst: a better understanding and new classification. Neurology 2001;56:220-227.

9. Sarnat HB. Embryology and malformations of the forebrain commissure. In: Sarnat HB, Curatolo P, eds. Malformations of the Nervous System. St. Louis: Elsevier; 2008:67.

10. Al-Gazali L, Bakalinova D. Autosomal recessive syndrome of macrocephaly, multiple epiphyseal dysplasia and distinctive facial appearance. Clin Dysmorphol 1998;7:177-184. 
11. Bayoumi R, Saar K, Lee YA, et al. Localisation of a gene for an autosomal recessive syndrome of macrocephaly, multiple epiphyseal dysplasia, and distinctive facies to chromosome 15q26. J Med Genet 2001;38:369-373.

12. Al-Gazali L, Hertecant J, Algawi K, El Teraifi H, Dattani $\mathrm{M}$. A new autosomal recessive syndrome of ocular colobomas, ichthyosis, brain malformations and endocrine abnormalities in an inbred Emirati family. Am J Med Genet A 2008; 146:813-819.

13. Chawalparit O, Wonglaksanapimon S, Songsaeng D, Saenanarong V. Reliability study of white matter rating scale for the dementia and disability in Thai Elderly Project. J Med Assoc Thai 2009;92:543-547.

14. Ay H, Furie KL, Singhal A, Smith WS, Sorensen AG, Koroshetz WJ. An evidence-based causative classification system for acute ischemic stroke. Ann Neurol 2005;58:688-697.

15. Goodyear PW, Bannister CM, Russell S, Rimmer S. Outcome in prenatally diagnosed fetal agenesis of the corpus callosum. Fetal Diagn Ther 2001;16:139-145.

16. Moutard ML, Kieffer V, Feingold J, et al. Agenesis of corpus callosum: prenatal diagnosis and prognosis. Childs Nerv Syst 2003;19:471-476.

17. Richards LJ, Plachez C, Ren T. Mechanisms regulating the development of the corpus callosum and its agenesis in mouse and human. Clin Genet 2004;66:276-289.
18. Ren T, Anderson A, Shen WB, et al. Imaging, anatomical, and molecular analysis of callosal formation in the developing human fetal brain. Anat Rec A Discov Mol Cell Evol Biol 2006;288:191-204.

19. Sherr EH. The ARX story (epilepsy, mental retardation, autism, and cerebral malformations): one gene leads to many phenotypes. Curr Opin Pediatr 2003;15:567-571.

20. Larbrisseau A, Vanasse M, Brochu P, Jasmin G. The Andermann syndrome: agenesis of the corpus callosum associated with mental retardation and progressive sensorimotor neuronopathy. Can J Neurol Sci 1984;11:257-261.

21. Aicardi J. Aicardi syndrome. Brain Dev 2005;27:164-171.

22. Sherr EH, Owen R, Albertson DG, et al. Genomic microarray analysis identifies candidate loci in patients with corpus callosum anomalies. Neurology 2005;65:1496-1498.

23. Boland E, Clayton-Smith J, Woo VG, et al. Mapping of deletion and translocation breakpoints in 1q44 implicates the serine/threonine kinase AKT3 in postnatal microcephaly and agenesis of the corpus callosum. Am J Hum Genet 2007;81:292-303.

24. Doco-Fenzy M, Mauran P, Lebrun JM, et al. Pure direct duplication (12) (q24.1-> q24.2) in a child with Marcus Gunn phenomenon and multiple congenital anomalies. Am J Med Genet A 2006;140:212-221.

25. Davila-Gutierrez G. Agenesis and dysgenesis of the corpus callosum. Semin Pediatr Neurol 2002;9:292-301.

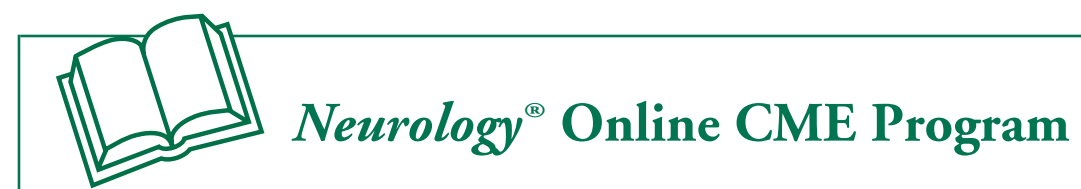

Earn CME while reading Neurology. This program is available only to online Neurology subscribers. Simply read the articles marked CME, go to www.neurology.org, and click on CME. This will provide all of the information necessary to get started. The American Academy of Neurology (AAN) is accredited by the Accreditation Council for Continuing Medical Education (ACCME) to sponsor continuing medical education for physicians. Neurology is planned and produced in accordance with the ACCME Essentials. For more information, contact AAN Member Services at 800-879-1960. 